\title{
Hemorrhoidopexy with the HemorPex System
}

\author{
M. Tagliabue $\cdot$ C. Cusumano $\cdot$ E. S. Kechoud $\cdot$ \\ D. Paternicò $\cdot$ M. Rasini $\cdot$ D. Carriero • \\ F. P. Paladino
}

Received: 19 November 2014 / Accepted: 20 December 2014/Published online: 23 January 2015

(C) Springer-Verlag Italia Srl 2015

\section{Introduction}

Transanal Doppler-guided hemorrhoidal artery ligation with mucopexy and hemorrhoidopexy with the HemorPex System (HPS) (ANGIOLOGICA B.M. S.r.l., Pavia, Italy) are minimally invasive non-excisional methods available to treat symptomatic hemorrhoids not responding to more conservative treatment. The HPS makes possible mucopexy and the ligature of superior hemorrhoidal artery branches, treating at the same time two important causes of hemorrhoids.

\section{Surgical technique}

HemorPex System, used for hemorrhoidopexy, consists of a rotating part, through which the sutures are placed, and a fixed part of the anoscope. This surgical instrument is provided with a fixed light focused on the surgical field (Fig. 1).

All but one of our patients were given spinal anesthesia and positioned in the prone decubitus or "jackknife"

\footnotetext{
M. Tagliabue $(\bowtie) \cdot$ C. Cusumano

Department of General Surgery, Ospedale Uboldo di Cernusco sul Naviglio, Azienda Ospedaliera di Melegnano, Via Uboldo 21, 20092 Cernusco sul Naviglio, Milan, Italy

e-mail: marta.tagliabue86@gmail.com

M. Tagliabue $\cdot$ C. Cusumano - F. P. Paladino

Residency Program in General Surgery, University of Milan,

Milan, Italy

E. S. Kechoud · D. Paternicò · M. Rasini · D. Carriero ·

F. P. Paladino

Department of General Surgery, Ospedale Uboldo di Cernusco sul Naviglio e Ospedale di Vaprio D’Adda, Azienda Ospedaliera di Melegnano, Milan, Italy
}

position. For one patient, we used the gynecological position and general anesthesia. As antibiotic prophylaxis, we used intravenous metronidazole (500 mg).

After introducing the operative device into the anal canal, 6 or 7 running sutures of polyglycolic acid 2/0 are positioned at 1,3,5,7,9,11 o'clock [1], the usual locations of the 6 main terminal branches of the superior rectal artery. We put another running suture (consisting of a glycolic acid and trimethylene carbonate copolymer) at 12 o'clock for severe prolapse (grades III and IV) (Fig. 2). The running sutures are placed in a clockwise direction starting from 11 o'clock. In each of the 6 or 7 positions, the continuous suture is characterized by 4 or 5 passages and corresponding knots through the wall so as to obtain a plication. During hemorrhoidopexy with the HPS, prolapse is exposed by retracting it outside the anal canal (Fig. 2b).

In this way, it is possible to lift up the sliding mucosa and submucosa for $3-4 \mathrm{~cm}$, about $2 \mathrm{~cm}$ above the dentate line, and close the arterial branches, like in the Doppler technique but without the aid of the Doppler signal.

To avoid local edema and promote the creation of a retractile scar, we usually used the spray function of the monopolar electric scalpel at the end of the hemorrhoidopexy (Fig. 2e).

In the long term, these sutures create a sort of "anchoring scar" between the mucosa, submucosa and the underlying musculature.

The average duration of the surgical procedure is $40 \mathrm{~min}$ (range 20-60 min).

\section{Results}

Between January 2010 and August 2014, we used HPS to treat 116 patients with symptomatic hemorrhoids at the 
surgical division of Uboldo Hospital, in Cernusco sul Naviglio, and Vaprio D'Adda Hospital (Milan). There were 83 males $(72 \%)$ and 33 females (28\%) with an average age of 51 years (range 22-81 years).

Eighty-eight patients had grade II (76\%), 21 grade III $(18 \%)$ and 7 grade IV (6\%) hemorrhoidal disease; 6 of these patients had recurrent hemorrhoids after having underwent prior surgical treatment, while 6 had an associated local pathology such as anal fissures or anal polyps that we treated at the time of surgery for the prolapse. For all patients, we recorded pain levels after surgery by means of the visual analog scale (VAS), obtaining a mean VAS

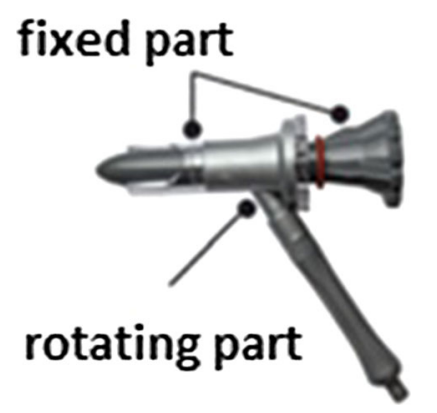

Fig. 1 HemorPex System (C) ANGIOLOGICA B.M. S.r.l., Pavia, Italy. Available at: http://www.hemorpexsystem.it/index.php?lang= $1 \&$ id=home score of $2( \pm 1)$ : The postoperative immediate pain was intense (VAS 6-10) in only 8 cases (11\%), medium in 5 cases $(7 \%)$ and light (VAS 1-3) in 95 cases (82\%). The postoperative pain was treated with paracetamol or nonsteroidal antiinflammatory drugs and at the time of the discharge, only oral painkillers were prescribed.

First defecation after the operation came spontaneously $24 \mathrm{~h}$ after the operation and at discharge, a diet regimen and stool softeners were prescribed for preventing constipation.

We never recorded immediate postoperative hemorrhage, but we registered 4 cases of severe bleeding (3.4\%) on day 7 and day 15 which needed surgical revision.

Only 1 patient had acute urinary retention after surgery. As for the presence of other symptoms, we observed anal and perianal edema in 2 cases (1.7\%) lasting 24-48 $\mathrm{h}$ after the operation and we recorded only 1 case of tenesmus in the first 3 months. One patient who had had prolapse associated with fissure developed a fistula.

At follow-up, we found hemorrhoids had recurred in 6 patients $(5.2 \%), 3$ of whom had recurrence for the second time (patients treated with HPS for recurrent prolapse), while the other 3 patients had not been operated on previously. The mean interval between the surgical procedure and the recurrence was 11.5 months.
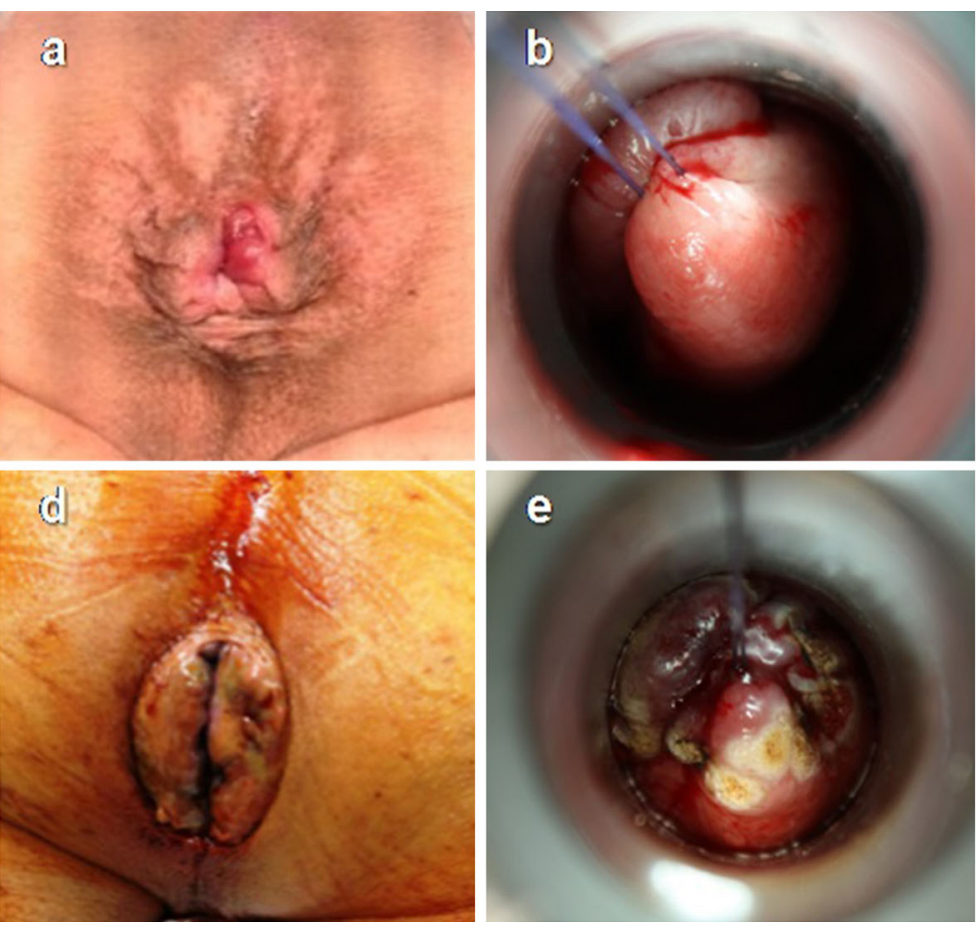


Fig. 2 Pre-, intra- and postoperative pictures. a Pre-treatment, b intraoperative treatment, and c post-treatment with HPS in a male patient with grade II hemorrhoids. b Plication of the mucosa and submucosa at 11 o'clock with the second step; the prolapse is highlighted with the traction exerted by the operator from inside the anal canal toward the outside. d Pre-treatment, e intraoperative hemorrhoidopexy and $\mathbf{f}$ post-treatment in a male patient with grade IV prolapse. e At the end of the plication, the spray function of the monopolar electric scalpel is used to minimize edema and congestion of the area just treated and create a retracting scar 


\section{Discussion}

HPS is quite a new minimally invasive technique for the treatment of hemorrhoids, and the literature on this topic is limited. We operated on grades II and III prolapse, for which HPS proved to be a good option in terms of percentage of complications (i.e., a $3.4 \%$ incidence of postoperative bleeding and a mean postoperative VAS of 2) and relapse, which are similar to the data found in the literature (postoperative bleeding between 0.5 and $4 \%$ ).

Postoperative bleeding was recorded in 3 cases on day 7 and in 1 case on day 15 . In 3 of these patients, bleeding was apparently due to the falling of the anchoring scar from mucosa and submucosa. In 1 patient, the bleeding seems to have been determined by sutures placed too deep with subsequent necrosis. Aiming at minimizing the postprocedure bleeding, we have made some changes in the surgical technique over the years: at the beginning, we used the monopolar scalpel with the coagulative function at the end of the pexis; recently, in order to avoid deep damage to the tissues and prevent postoperative bleeding, we preferred the spray function.

In the literature, recurrent prolapse is not considered a contraindication to hemorrhoidopexy, even when performed for the second time. We treated 6 patients with a recurrent prolapse: At follow-up, 3 of them still had symptoms related to hemorrhoids. This result seems to show that HPS is not the best treatment for recurrence, but these considerations need to be validated with bigger studies.
Hemorrhoidopexy is not recommended in grade IV prolapse in the literature [2]. We treated 7 patients with grade IV prolapse and 2 of them with a recurrent prolapse. Three of these 7 patients had postoperative complications: 1 patient developed bleeding on day 7 which required surgical revision; 2 patients experienced recurrent prolapse, after a mean time period of 6 months. Because of the limited data and short follow-up, we cannot state a significant trend, but our experience seems to confirm the literature data.

\section{Conclusions}

Hemorrhoidopexy with HPS is a valid and safe treatment for II and III degree prolapses, offering satisfying results in terms of postoperative bleeding and recurrence of symptoms and minimal patient discomfort postoperatively.

Conflict of interest None.

\section{References}

1. Basile M, Di Resta V, Ranieri E (2014) Transanalanopexy with HemorPex System (HPS) is effective in treating grade II and III haemorrhoids: long term results. http://www.hemorpexsystem.it/ pubblicazioni/Articolo-HPS-Dr-Basile.pdf

2. Boccasanta P, Capretti PG, Venturi M et al (2001) Randomised controlled trial between stapled circumferential mucosectomy and conventional circular hemorrhoidectomy in advanced hemorrhoids with external mucosal prolapse. Am J Surg 182:64-68 